\title{
Commentary on the 2008 WHO classification of mature T- and NK-cell neoplasms
}

\author{
Megan S. Lim • Laurence de Leval • \\ Leticia Quintanilla-Martinez
}

Received: 29 April 2009/Accepted: 1 May 2009/Published online: 27 June 2009

(C) Springer-Verlag 2009

\begin{abstract}
In 2008, the World Health Organization (WHO) published a revised and updated edition of the classification of tumors of the hematopoietic and lymphoid tissues. The aims of the fourth edition of the WHO classification was to incorporate new scientific and clinical information in order to refine diagnostic criteria for previously described neoplasms and to introduce newly recognized disease entities. The recognition that T-cell lymphomas are related to the innate and adaptive immune system, as well as enhanced understanding of other T-cell subsets, such as the regulatory T-cell and follicular helper T-cells, has contributed to our understanding of the morphologic, histologic, and immunophenotypic features of T- and NK-cell neoplasms. The purpose of this review is to highlight major changes in the classification of $\mathrm{T}$ - and $\mathrm{NK}$-cell neoplasms and to explain the rationale for these changes.
\end{abstract}

Keywords Mature T-cell neoplasm . Mature NK-cell neoplasm · 2008 WHO classification

M. S. Lim

Department of Pathology,

University of Michigan Medical School,

Ann Arbor, MI, USA

L. de Leval

CHU Sart Tilman, University of Liege,

Liege, Belgium

L. Quintanilla-Martinez $(\square)$

Institute of Pathology, Eberhard-Karls-University of Tübingen,

Liebermeisterstrasse 8,

72076 Tübingen, Germany

e-mail: leticia.quintanilla-fend@med.uni-tuebingen.de

\section{Introduction}

The 2008 World Health Organization (WHO) classification of mature thymus (T)- and natural killer (NK)-cell neoplasms provide clarification regarding diagnostic criteria, etiologic insights, and prognostic information for several new/provisional entities [1]. The recognition that T-cell lymphomas are related to the innate and adaptive immune system, as well as enhanced understanding of other T-cell subsets, such as the regulatory T-cell and follicular helper T-cells (TFH), has contributed to our understanding of the morphologic, histologic, and immunophenotypic features of T- and NK-cell neoplasms. New insights regarding immunophenotypic features of large granular lymphocytes and NK-cells have provided better flow cytometric tools for the diagnosis of T-cell large granular lymphocytic leukemia (TLGL). The broad clinical spectrum of NK-cell neoplasms is now recognized with the new provisional entity encompassed by chronic lymphoproliferative disorder of NKcells. The importance of the role of Epstein-Barr virus (EBV) in two T-cell lymphoproliferative disorders of childhood has resulted in the addition of systemic EBVpositive T-cell lymphoproliferative disease of childhood and hydroa vacciniforme-like lymphoma as new entities. In enteropathy-associated T-cell lymphoma (EATL), two different types are now recognized: the classical form and the monomorphic type. Additionally, a precursor lesion is recognized as intraepithelial T-cell lymphoma or EATL in situ. Subcutaneous panniculitis-like T-cell lymphoma (SPTCL) is now considered to be a neoplasia of mature cytotoxic $\alpha \beta$ T-cells. In contrast to the previous WHO classification, cases expressing the $\gamma \delta$ T-cell receptor (TCR) are excluded and now reclassified as primary cutaneous $\gamma \delta$ T-cell lymphomas. The staging system for mycosis fungoides (MF) has been better defined. Further- 
more, the histologic and immunophenotypic heterogeneity within the T-cell lymphomas with cutaneous presentation is recognized in the 2008 WHO classification resulting in several provisional entities including primary cutaneous CD8+ aggressive lymphoma and primary cutaneous CD4+ small/medium T-cell lymphoma. Of note, blastic NK-cell lymphoma is now considered to be a tumor derived from a plasmacytoid dendritic cell and is included in the myeloid neoplasms. Angioimmunoblastic T-cell lymphoma (AITL) has emerged as a distinctive subtype of peripheral T-cell lymphoma (PTCL) derived from follicular T helper cells. Anaplastic lymphoma kinase (ALK)-positive and ALKnegative cases have been separated based on clinical and pathogenetic data.

\section{T-cell and NK-cell neoplasms with leukemic presentation}

Mature T-cell and NK-cell neoplasms with a predominant leukemic or disseminated clinical presentation are represented by the following entities: T-cell prolymphocytic leukemia (T-PLL), T-LGL, chronic lymphoproliferative disorders of NK-cells (new entity), aggressive NK-cell leukemia, adult T-cell leukemia/lymphoma (ATLL), and Sezary syndrome (SS; Table 1).

T-cell prolymphocytic leukemia (T-PLL)

No significant changes have been made. The 2008 WHO classification emphasizes the role of TCL1 immunohistochemistry, which may be helpful in identifying residual TPLL on bone marrow sections or lymph nodes after therapy.
It is important to highlight that TCL1 can be expressed in reactive and neoplastic B-cells and other T-cell neoplasms.

T-cell large granular lymphocytic leukemia (T-LGL)

The updated 2008 WHO classification provides additional insights into the etiology of this rare and heterogenous disorder. T-LGL represents a clonal population of cytotoxic effector T-cells. The frequent association with autoimmune disorders such as rheumatoid arthritis supports the notion that these clones arise in setting of sustained immune stimulation. Additionally, clonal expansions of T-LGL are observed following allogeneic bone marrow transplantation and in association with low-grade B-cell lymphomas. These are thought to reflect a restricted T-cell repertoire or a type of host response.

New insights into the expression of the CD94/NKG2 and killer immunoglobulin-like receptors (KIR) families of NK-associated major histocompatibility complex-class I receptors in T-LGLs are provided in the updated 2008 WHO classification. More than $50 \%$ of T-LGL leukemias express CD94/NKG2 and KIR family receptors. The uniform expression of a single isoform of KIR can serve as a surrogate for clonality.

\section{Chronic lymphoproliferative disorder of NK-cells}

Chronic lymphoproliferative disorder of NK-cells were previously designated as chronic NK-cell lymphocytosis, chronic NK-large granular lymphocyte lymphoproliferative disorder, and NK-cell LGL lymphocytosis, all of which reflected the variable and heterogeneous neoplastic potential of the proliferations. They are rare and are characterized

Table 1 Summary of the categories of T-cell and NK-cell neoplasms with a predominant leukemic presentation within the 2001 and 2008 WHO classification

\begin{tabular}{|c|c|c|}
\hline 2001 WHO classification & 2008 WHO classification & Comments \\
\hline T-cell prolymphocytic leukemia & T-cell prolymphocytic leukemia & No changes \\
\hline \multirow[t]{2}{*}{$\begin{array}{l}\text { T-cell large granular lymphocytic } \\
\text { leukemia }\end{array}$} & $\begin{array}{l}\text { T-cell large granular lymphocytic } \\
\text { leukemia }\end{array}$ & $\begin{array}{l}\text { New insights into etiology } \\
\text { New insights into the expression of the CD94/NKG2 and KIR } \\
\text { KIR expression as surrogate marker for clonality }\end{array}$ \\
\hline & $\begin{array}{l}\text { Chronic lymphoproliferative } \\
\text { disorder of NK-cells }\end{array}$ & $\begin{array}{l}\text { New provisional entity based on the need to } \\
\text { distinguish from aggressive NK-cell leukemia }\end{array}$ \\
\hline Aggressive NK-cell leukemia & Aggressive NK-cell leukemia & \\
\hline Adult T-cell leukemia/lymphoma & Adult T-cell leukemia/lymphoma & $\begin{array}{l}\text { Diagnostic criteria for clinical subtypes } \\
\text { Neoplastic counterpart for CD4+CD25+FOXP3+ } \\
\text { regulatory T-cells }\end{array}$ \\
\hline Sezary syndrome & Sezary syndrome & $\begin{array}{l}\text { Diagnostic criteria } \\
\text { New immunophenotypic markers, cutaneous } \\
\text { lymphocyte antigen }+ \text {, skin-homing receptor } \\
\text { CCR4+, and lack CD7 and CD26 }\end{array}$ \\
\hline
\end{tabular}


by a persistent ( $>6$ months duration) increase in peripheral blood NK-cells $(>2 \times 109 / \mathrm{L})$ without a clearly identified cause. Reactive and neoplastic proliferations are difficult to distinguish, and it is currently a provision entity within the WHO classification. NK-cells are negative for surface CD3 but positive for cytoplasmic $\mathrm{CD} 3 \varepsilon$ (the antigen recognized by the paraffin CD3 antibody); CD16 with weak CD56 expression is frequently observed. Markers of cytotoxic Tcells such as TIA1, granzyme $B$, and granzyme $M$ are positive. The expression of $\mathrm{CD} 2, \mathrm{CD} 7$, and $\mathrm{CD} 57$ may be diminished or absent while aberrant coexpression of CD5 and CD8 can be seen. Expression of the KIR family of NKcell receptors is either restricted to one isoform or completely lacking. At the molecular level, there are no rearrangements of the immunoglobulin and TCR genes. The karyotype is normal in most cases. Clonality assays have demonstrated that a subset of cases is clonal. EBV is negative in contrast to aggressive NK-cell leukemia.

Aggressive NK-cell leukemia

Expression of Fas ligand by the neoplastic cells with elevated levels in the serum is a new observation. Notably, a clear distinction between aggressive NK-cell leukemia and chronic lymphoproliferative disorders of NK-cells is made in the current WHO classification.

\section{Adult T-cell leukemia/lymphoma (ATLL)}

The 2008 WHO classification provides a diagnostic criteria for clinical subtypes of ATLL which is correlated with clinical outcome. The three clinical subtypes are smoldering, chronic, and acute. While four clinical variants are recognized, diagnostic criteria for only three of these are outlined in the updated classification. Furthermore, it is unclear whether the terms "variants" and "subtypes" are used interchangeably. The postulated normal counterpart of ATLL is considered to be peripheral blood CD4+ Tcells which show an immunophenotype closest to the CD4+CD25+FOXP3+ regulatory T-cells.

Sezary syndrome (SS)

A distinct and separate section has been created to discuss SS in the 2008 WHO classification. Diagnostic criteria for SS are outlined which include the presence of one or more of the following: an absolute Sezary cell count of at least 1,000 cells per $\mathrm{mm}^{3}$, an expanded CD4+ T-cell population resulting in a CD4/CD8 ratio of more than 10 , and/or loss of one or more T-cell antigens. The immunophenotype of Sezary cells is better defined in the updated classification. In addition to $\mathrm{CD} 2, \mathrm{CD} 3, \mathrm{CD} 4$, and $\mathrm{CD} 5$, they express cutaneous lymphocyte antigen (CLA) and the skin-homing receptor
CCR4 and lack CD7 and CD26. In addition, the expression of T-plastin is greatly increased in Sezary cells relative to normal helper T-cells. Recent studies using molecular cytogenetic techniques such as M-FISH and comparative genomic hybridization techniques have shown a high rate of unbalanced translocations and associated deletions involving chromosome 1p, 6q, 10q, 17p, and 19 suggesting the role of genetic instability. Other molecular abnormalities include inactivation of TP53, $p 16^{I N K 4 a}$, and amplification of JUNB.

\section{EBV-positive T-cell lymphoproliferative disorders of childhood}

In the 2008 WHO classification, two new major types of EBV-associated T-cell lymphoproliferative disorders affecting pediatric population have been incorporated: systemic EBV-positive T-cell lymphoproliferative disease of childhood and hydroa vacciniforme-like lymphoma. Both of them occur predominantly in Asians and in Native Americans from Central and South America and Mexico.

Systemic EBV-positive T-cell lymphoproliferative disease of childhood This is a life-threatening illness of children and young adults characterized by a clonal proliferation of EBV-infected T-cells with an activated cytotoxic phenotype. It usually occurs shortly after primary acute EBV infection in previously healthy patients or in the setting of chronic active EBV infection (CAEBV). It has a rapid progression with multiple organ failure, sepsis, and death, usually from days to weeks. The most frequent sites of involvements are liver and spleen followed by lymph nodes, bone marrow, skin, and lung. The most typical phenotype of the tumor cells is $\mathrm{CD} 2+\mathrm{CD} 3+\mathrm{CD} 56-$ and positive for cytotoxic proteins. Most cases secondary to acute primary EBV infection are $\mathrm{CD} 8+$; whereas, cases in the setting of severe CAEBV are $\mathrm{CD} 4+$. EBV is always positive. The tumor cells have monoclonaly rearranged TCR genes.

Hydroa vacciniforme-like lymphoma This is an EBVpositive cutaneous T-cell lymphoma (CTCL) occurring in children and associated with sun sensitivity. This condition affects primarily sun-exposed skin, in particular, the face. These cases have usually a CD8+ cytotoxic phenotype. Most cases have clonal rearrangement of the TCR genes. EBVencoded RNA (EBER) in situ hybridization is universally positive, but LMP1 is generally negative. It is still not clear whether severe mosquito-bite allergy, which is of NK derivation and EBV-associated, is part of hydroa vacciniforme-like lymphoma or a distinctive entity within the spectrum of EBV-associated disorders. Both disorders are considered part of the spectrum of severe CAEBV, with a broad spectrum of clinical aggressiveness. 


\section{T-cell and NK-cell neoplasms with extranodal presentation}

Extranodal T-cell and NK-cell neoplasms in the 2008 WHO includes extranodal NK/T-cell lymphoma, nasal type; EATL; hepatosplenic T-cell lymphoma; and SPTCL (Table 2).

Extranodal NK/T-cell lymphoma, nasal type

No significant changes have been made. The 2008 WHO emphasizes that although extranodal NK/T-cell lymphoma, nasal type occurs predominantly in the upper aerodigestive tract, extranasal involvement is not infrequent (extranasal NK/T-cell lymphomas), and both presentations are EBVassociated. By definition, the neoplastic cells show CD56 expression and cytoplasmic CD $3 \varepsilon$ with negative surface CD3, although in rare cases, CD56 might be weak or even negative. EBER is positive virtually in every neoplastic cell and is a prerequisite for the diagnosis. LMP1 is often negative. Cytotoxic molecules (granzyme B, perforin, and TIA1) are positive. Other T- and NK-cell markers such as CD4, CD5, CD2, CD16, CD57, CD43, CD45RO, TCR $\gamma$, and $\beta F 1$ are negative. Occasional cases are positive for CD8, CD7, or CD30. Recent comparative genomic hybridization studies showed some recurrent chromosomal alterations. A proportion of cases have partial deletion of FAS gene, mutations of TP53, $\beta$-catenin, $K-R A S$, or $C$-KIT gene, but the significance of all these alterations is still unclear. It is important to consider in the differential diagnosis a spectrum of EBV-associated T-cell or NK-cell lymphoproliferative disorders, including CAEBV, and the new recognized group of systemic EBV+ T-cell lymphoproliferative disease of childhood.
Enteropathy-associated T-cell lymphoma (EATL)

The most important changes in the 2008 WHO classification are the recognition of two different types of EATL and its genetic changes. EATL-classical form is the most frequent type (80-90\%) and is associated with celiac disease. The degree of associated enteropathy is highly variable; some patients have a long history of celiac disease, and some are diagnosed in the same episode in which the lymphoma is diagnosed. Although the cytological appearance is variable, most cases are composed of large lymphoid cells often with an inflammatory background. The immunophenotype of the tumor cells is $\mathrm{CD} 3+$, CD5-, CD7+, CD8- $(20 \%+)$, CD4-, CD103+, TCR $\beta+/-$, contains cytotoxic granules-associated proteins and, in almost all cases, a proportion of the tumor cells express CD30. The intraepithelial lymphocytes (IEL) in the adjacent enteropathic mucosa may show an abnormal phenotype, usually CD3+, CD5-, CD8-, and CD4-.

EATL-type II or monomorphic type represents $10-20 \%$ of all EATL cases. This variant may occur sporadically, without risk factors for celiac disease, suggesting that the monomorphic variant represents a distinct disease entity. This variant is composed of monomorphic medium-sized cells with a distinctive immunophenotype. The tumor cells are CD3+, CD4-, CD8+, CD56+, and $\mathrm{TCR} \beta+$. Interestingly, both forms of EATL are characterized by complex segmental amplifications of the 9q31.3-qter chromosome region in 58$70 \%$ of the cases or by deletions in $16 q 12.1$. Additionally, the classical form of EATL shows chromosomal gains in $1 \mathrm{q}$ and 5q; whereas, the monomorphic variant has 8q24 (MYC) amplifications. Importantly, both classical EATL and the monomorphic form have a similar clinical course.

Table 2 Summary of the categories of T-cell and NK-cell neoplasms with a predominant extranodal presentation within the 2001 and 2008 WHO classification

\begin{tabular}{|c|c|c|}
\hline 2001 WHO classification & 2008 WHO classification & Comments \\
\hline $\begin{array}{l}\text { Extranodal NK/T-cell lymphoma, } \\
\text { nasal type }\end{array}$ & $\begin{array}{l}\text { Extranodal NK/T-cell lymphoma, } \\
\text { nasal type }\end{array}$ & No major changes \\
\hline Enteropathy-associated T-cell lymphoma & $\begin{array}{l}\text { Enteropathy-associated } \\
\text { T-cell lymphoma (EATL) }\end{array}$ & $\begin{array}{l}\text { Two forms are recognized: EATL-classical form is associated } \\
\text { to celiac disease, CD3+,CD4-, CD8-, and CD56- and } \\
\text { EATL-type II or monomorphic type, CD8+, CD56+, and CD4- } \\
\text { Common genetic alterations: amplification of 9q31.3 or deletion } \\
\text { of } 16 \mathrm{q} 21 \\
\text { Precursor lesions are recognized EATL in situ/intraepithelial } \\
\text { T-cell lymphoma }\end{array}$ \\
\hline Hepatosplenic T-cell lymphoma & Hepatosplenic T-cell lymphoma & No major changes \\
\hline $\begin{array}{l}\text { Subcutaneous panniculitis-like } \\
\text { T-cell lymphoma }\end{array}$ & $\begin{array}{l}\text { Subcutaneous panniculitis-like } \\
\text { T-cell lymphoma }\end{array}$ & $\begin{array}{l}\text { Includes only the } \alpha / \beta \text { type } \\
\text { Twenty percent associated to autoimmune disease, mostly } \\
\text { systemic lupus erythematosus }\end{array}$ \\
\hline
\end{tabular}


The 2008 WHO classification emphasizes that refractory celiac disease (RCD) in which the IEL show downregulation of CD8 and monoclonal T-cell rearrangements, which usually is the same monoclonal TCR gene rearrangement as the subsequent developed T-cell lymphoma, represents most probably a precursor lesion and, therefore, can be considered as examples of intraepithelial T-cell lymphoma or EATL in situ. The monomorphic form of EATL may also be preceded by RCD in which the IEL immunophenotype is similar to the neoplastic cells of the subsequent lymphoma, namely, CD8+CD56+.

Hepatosplenic T-cell lymphoma (HSTL)

No significant changes have been made. Aberrant expression of multiple KIR isoforms along with dim or absent CD94 is a new observation. The 2008 WHO classification stresses the fact that $20 \%$ of HSTL arise in the setting of chronic immunosuppression, most commonly after solid organ transplantation, however, may also occur in children treated with azathioprine and infliximab for Crohn's disease.

\section{Subcutaneous panniculitis-like T-cell lymphoma (SPTCL)}

The most important change in the 2008 WHO classification is that the cases expressing $\gamma \delta$ TCR are excluded from SPTCL and are now reclassified as primary cutaneous $\gamma \delta$ T-cell lymphoma (see below). The new 2008 WHO classification also emphasizes the association of SPTCL with autoimmune disorders that can be found in up to $20 \%$ of the cases. The most frequent association is with systemic lupus erythematosus. Furthermore, SPTCL may show overlapping features with lupus profundus panniculitis. The immunophenotype is of a mature $\alpha \beta$ T-cell with expression of $\mathrm{CD} 8$, cytotoxic associated proteins, and $\beta F 1$. In contrast to cutaneous $\gamma \delta$ T-cell lymphoma, the tumor cells in SPTCL do not express CD56, hemophagocytic syndrome is seen only in $15-20 \%$ of the cases, and SPTCL has a much better prognosis. EBV virus is always absent.

\section{T-cell neoplasms with nodal presentation}

Mature T-cell neoplasms with a predominantly nodal presentation include the following entities: AITL; anaplastic large cell lymphoma (ALCL), ALK-positive; ALCL, ALKnegative; and peripheral T-cell lymphoma (PTCL), not otherwise specified (NOS; Table 3).

\section{Angioimmunoblastic T-cell lymphoma (AITL)}

The most significant advances in respect with this disease entity are (1) the demonstration of CD10 expression by at least a fraction of the neoplastic cells, in most cases, a feature very helpful for diagnostic purposes, and (2) the recent identification of the cellular origin of the neoplastic T-cell of AITL. Several studies have indeed provided convergent evidence that AITL tumor cells have immunophenotypic and molecular features overlapping with those of a specific subset of CD4+ helper T-cells, namely, TFH, normally located in the light zone of germinal centers where they exert a functional help for follicular B-cell differentiation. This specific cellular derivation likely explains several of the peculiar patholobiological features inherent to this disease, for example, the expansion of B-cells and hypergammaglobulinemia and the striking proliferation of follicular dendritic cells. Moreover, importantly, several phenotypic markers of normal TFH cells including the CXCL13 chemokine and program death-1 (PD-1, a member of the CD28 costimulatory membrane receptor family) have been validated in AITL neoplastic cells and represent novel diagnostic markers for the identification of AITL tumor cells in lymph nodes and in cases of extranodal dissemination of the disease.

Table 3 Summary of the categories of T-cell neoplasms with a predominantly nodal presentation, as listed in the 2001 and 2008 WHO classifications

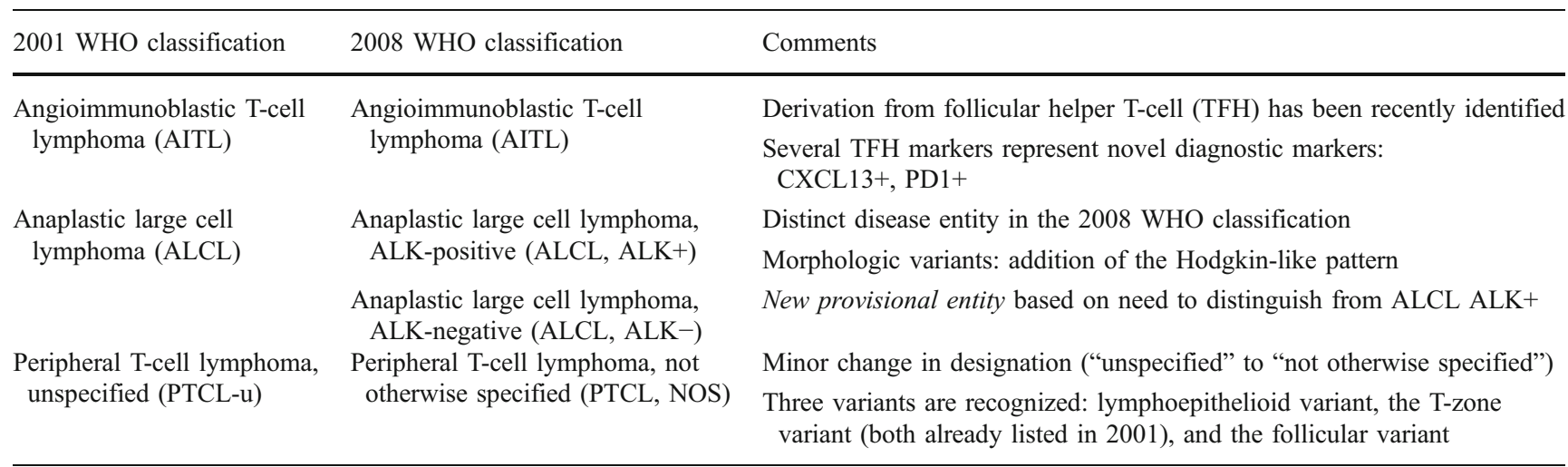


Anaplastic large cell lymphoma, ALK-positive (ALCL, ALK + )

The definition of ALCL remains that of a T-cell lymphoma composed of large cells with abundant cytoplasm and pleomorphic horseshoe-shaped nuclei including a proportion of so-called "hallmark cells" (with a paranuclear eosinophilic region) and characterized by strong CD30 expression. The major change introduced in the $2008 \mathrm{WHO}$ classification is the segregation of systemic ALCL into two separate entities depending on the presence or absence of $A L K$ gene rearrangement and upregulation of ALK protein. In the 2001 WHO classification, both ALK-positive and ALK-negative ALCL were considered as two variants of the same entity. ALCL, ALK-positive represents a welldelineated entity defined by a characteristic genetic alteration consisting of rearrangement of the $A L K$ gene. Remarkably, this entity is associated with an overall good prognosis, superior to that of any other nodal T-cell lymphoma entity. The morphologic spectrum of ALKpositive ALCL is broad and has been expanded to include the recently described "Hodgkin-like" pattern, mimicking nodular sclerosis Hodgkin lymphoma. The lymphohistiocytic and small cell patterns, which are the most common morphologic variants, tend to be associated with a lesser favorable outcome. Conversely, the genetic heterogeneity of ALK translocations (to various partner genes), which dictates the subcellular distribution of ALK fusion proteins, bears no correlation with the clinical features nor with the morphology.

Anaplastic large cell lymphoma, ALK-negative (ALCL, ALK-)

ALCL, ALK-negative is introduced in the 2008 WHO classification as a provisional entity. It is defined as a CD30+ large cell neoplasm that by morphology is indistinguishable from the common pattern of ALCL, ALK-positive (i.e., contains hallmark cells) but lacks ALK gene rearrangement and is negative for ALK protein expression. Other immunophenotypic features are similar to those of ALCL, ALK-positive, but the expression of pan-Tcell antigens tends to be more frequent, while the expression of cytotoxic markers and of epithelial membrane antigen (EMA) is less common. As the morphological variants of ALCL cannot reliably be identified without ALK expression, ALK-negative ALCL comprises exclusively cases with classical morphology. As the defining criteria of this entity are in part subject to inter-observer variability, the borders of this entity are not well defined, and in particular, there is some overlap with a subset of PTCL, NOS showing CD30 expression. Clinically, ALCL, ALKnegative has a prognosis much worse than ALK-positive ALCL but an outcome superior to that of PTCL, NOS.
Peripheral T-cell lymphoma, not otherwise specified (PTCL, NOS)

The category of the PTCL, unspecified (PTCL-u) in the 2001 WHO classification is now designated as "PTCL, not otherwise specified" (PTCL, NOS), an appellation reflecting the expectations for better specification or subdivisions in the future. This category defined by exclusion encompasses all mature T-cell neoplasms lacking specific features that would allow categorization within any of the better-defined "specific" subtypes of PTCL described in the WHO classification. PTCL, NOS often affects lymph nodes, but presentation in extranodal sites, such as the skin, is also frequently encountered.

The two variants of PTCL, NOS listed in the 2001 WHO classification are maintained in the 2008 WHO edition, i.e., the lymphoepithelioid variant, also known as Lennert's lymphoma, which is derived from CD8+ T-cells in most instances and the T-zone variant, characterized by a perifollicular growth pattern and a small cell morphology, both of which may be associated with a more indolent clinical course. A third variant more recently describedfollicular PTCL - has been added to the current classification. This peculiar and rare form of PTCL, NOS refers to a pattern of growth intimately related to follicular structures, which may mimic follicular lymphoma, exhibit features suggestive of marginal zone lymphomas or involve mantle zones. Interestingly, follicular PTCL exhibits a TFH phenotype, questioning its possible relationship to AITL.

\section{T-cell neoplasms with cutaneous presentation}

Cutaneous T-cell lymphomas (CTCLs) in the 2008 WHO include the following entities: MF; SS; primary cutaneous CD30-positive T-cell lymphoproliferative disorders, including primary cutaneous ALCL and lymphomatoid papulosis (LyP); and the new group of primary cutaneous PTCLs, rare subtypes, which includes primary cutaneous gammadelta T-cell lymphoma (PCGD-TCL), primary cutaneous CD8-positive aggressive epidermotropic cytotoxic T-cell lymphoma, and primary cutaneous CD4-positive small/ medium T-cell lymphoma. A disease that was previously considered to be of NK-derivation, the blastic NK-cell lymphoma, has changed its name to blastic plasmacytoid dendritic cell neoplasm (BPDC), and it is now classified within the myeloid neoplasms (Table 4).

Mycosis fungoides (MF)

The most significant changes in this disease are the defining criteria for clinical staging and the histopatho- 
Table 4 Summary of the category of T-cell lymphomas with a predominant cutaneous involvement within the 2001 and 2008 WHO classification

\begin{tabular}{|c|c|c|}
\hline 2001 WHO classification & 2008 WHO classification & Comments \\
\hline Mycosis fungoides & Mycosis fungoides & $\begin{array}{l}\text { Better-defined staging system } \\
\text { Constitutive activation of Stat3 } \\
\text { TNF in the tumorigenesis of MF } \\
\text { CD8+ in pediatric MF }\end{array}$ \\
\hline Sezary syndrome & Sezary syndrome & $\begin{array}{l}\text { Better-defined diagnostic criteria } \\
\text { New immunophenotypic markers, cutaneous } \\
\text { lymphocyte antigen+, skin-homing receptor } \\
\text { CCR4+, and lack CD7 and CD26 }\end{array}$ \\
\hline $\begin{array}{l}\text { Primary cutaneous anaplastic large } \\
\text { cell lymphoma (C-ALCL) }\end{array}$ & $\begin{array}{l}\text { Primary cutaneous anaplastic } \\
\text { large cell lymphoma (C-ALCL) }\end{array}$ & $\begin{array}{l}\text { Rare cases CD8+ } \\
\text { Rare coexpression of CD56+ }\end{array}$ \\
\hline Lymphomatoid papulosis & Lymphomatoid papulosis & $\begin{array}{l}\text { Three histologic subtypes are recognized }(\mathrm{A}, \mathrm{B} \text {, and } \mathrm{C}) \\
\text { Rare cases of } \mathrm{CD} 8+\text { and NK phenotype }\end{array}$ \\
\hline \multirow[t]{3}{*}{$\begin{array}{l}\text { Subcutaneous panniculitis-like } \\
\text { T-cell lymphoma with a } \gamma / \delta \\
\text { phenotype }\end{array}$} & $\begin{array}{l}\text { Primary cutaneous gamma-delta } \\
\text { T-cell lymphoma }\end{array}$ & $\begin{array}{l}\text { Three histologic patterns: epidermotropic, dermal, } \\
\text { and subcutaneous } \\
\mathrm{CD} 3+, \mathrm{CD} 56+\mathrm{TCR} \delta+\text { and EBV negative CD4-,CD8-, } \\
\text { and } \beta \mathrm{F} 1-\end{array}$ \\
\hline & $\begin{array}{l}\text { Primary cutaneous CD8+ aggressive } \\
\text { epidermotropic cytotoxic T-cell } \\
\text { lymphoma }\end{array}$ & $\begin{array}{l}\text { Provisional entity } \\
\text { Disseminates to visceral sites } \\
\text { Cell of origin: CD } 8+\text { cytotoxic T-cell of } \alpha / \beta \text { subtype } \\
\text { Aggressive disease }\end{array}$ \\
\hline & $\begin{array}{l}\text { Primary cutaneous CD4+ small/medium } \\
\text { T-cell lymphoma }\end{array}$ & $\begin{array}{l}\text { Provisional entity } \\
\text { Solitary skin lesion on the head and neck } \\
\text { Cell of origin: skin-homing CD4+ T-cell } \\
\text { Favorable prognosis }\end{array}$ \\
\hline Blastic NK-cell lymphoma & $\begin{array}{l}\text { Blastic plasmocytoid dendritic } \\
\text { cell neoplasm }\end{array}$ & $\begin{array}{l}\text { Included now with myeloid neoplasias } \\
\text { CD56+TdT+CD4, CD43, TCL1, and CD123 }\end{array}$ \\
\hline
\end{tabular}

logical staging for "clinically abnormal" lymph nodes. The purpose of revising the staging and classification system was to incorporate all the steady advances in the areas of molecular biology, immunohistochemistry, and imaging as well as new data on prognostic variables in MF. The updated International Society for Cutaneous Lymphomas/European Organization of Research and Treatment of Cancer (ISCL/EORTC) revision to the $\mathrm{N}$ (node) classification was incorporated into the 2008 WHO classification. This staging system incorporated the previous National Cancer Institute and Dutch lymph node staging systems. This classification eliminates biopsies of lymph nodes for staging purposes, unless they are "clinically abnormal". The definition of "clinically abnormal" lymph nodes refers to lymph nodes larger than $1.5 \mathrm{~cm}$. There are three categories recognized. The N1 category includes dermatopathic lymphadenopathy and the presence of occasional atypical lymphocytes with clusters of three to six cells. The N2 category includes dermatopathic lymphadenopathy with early involvement with scattered atypical cerebriform mononuclear cells, defined as cells with diameter larger than
7.5 $\mu \mathrm{m}$. The architecture here is still preserved. The N3 category includes overt involvement of the lymph nodes with both, partial, or complete architecture effacement. N3 lymph nodes may simulate PTCLs, NOS or Hodgkin lymphoma.

The clinical staging system was better defined. Stage I includes disease confined to the skin with patches, papules, and or plaques with no "clinically abnormal" lymph nodes $(<10 \%$ skin surface, stage IA; $>10 \%$, stage IB). Stage II includes skin involvement associated with early (N1-N2) lymph node involvement (stage IIA) or one or more skin tumors $(>1 \mathrm{~cm}$, stage IIB). Stage III includes skin involvement with erythroderma, N1-N2 lymph node involvement, and absent or low blood tumor burden $(<1,000$ / $\mathrm{ml}$ circulating Sezary cells). Stage IV includes high blood tumor burden $(>1,000 / \mathrm{ml}$ circulating Sezary cells) and/or extensive lymph node involvement (N3) or visceral involvement.

New studies have identified the constitutive activation of the transcription factor STAT3 and the inactivation of $C D K 2 A / p 16 i n k 4$ and PTEN. Both changes might be 
associated with disease progression. New gene expression profiling studies have shown tumor necrosis factor (TNF) anti-apoptotic pathway activation in the tumorigenesis of MF. Interestingly, a CD8+ MF has been incorporated in the 2008 WHO, which has been reported more commonly in pediatric population.

Primary cutaneous CD30-positive T-cell

lymphoproliferative disorders

Primary cutaneous $\mathrm{CD} 30+$ lymphoproliferative disorders are the second most common group of CTCLs, accounting for approximately $30 \%$ of the cases. This group includes primary cutaneous (C)-ALCL, LyP, and borderline cases.

Primary cutaneous anaplastic large cell lymphoma (CALCL) No significant changes have been made. The 2008 WHO classification emphasizes the role of immunophenotype to differentiate cutaneous and systemic ALCL. CALCL neoplastic cells show an activated CD4+ T-cell phenotype with frequent expression of cytotoxic proteins. It is important to highlight that some cases $(<5 \%)$ have a CD8+ T-cell phenotype. Unlike systemic ALCL, most CALCLs express the CLA but do not express EMA or ALK. Coexpression of CD56 is rarely observed, and its expression is not associated with unfavorable prognosis.

Lymphomatoid papulosis (LyP) No significant changes have been made. The 2008 WHO classification recognizes three histological subtypes (Types A, B, and C), which represent a spectrum of the disease with overlapping features. The type A and B lesions remained as previously defined. The type $\mathrm{C}$ lesions are composed of a monotonous population or large clusters of large CD30positive T-cells with relative few admixed inflammatory cells. The immunophenotype of Type A and C lesions is identical to the tumor cells in C-ALCL. The tumor cells in LyP type B lesions have a CD3+, CD4+, CD8phenotype and do not express CD30 antigen. Importantly, rare cases of LyP have been reported to have a $\mathrm{CD} 8+$ or an NK-cell phenotype.

Primary cutaneous peripheral T-cell lymphomas, rare subtypes

This group is composed of three new entities in the 2008 WHO classification, two of them are still considered as provisional entities.

Primary cutaneous gamma-delta T-cell lymphoma These cases were included in the previous WHO classification in the group of SPTCL with a gamma/delta phenotype. However, due to the extensive involvement of the subcutaneous tissue, as well as the dermis and epidermis, these cases are now classified in the 2008 WHO classification as part of the primary cutaneous PTCLs. PCGD-TCL is a lymphoma composed of a clonal proliferation of mature, activated $\gamma / \delta$ T-cells with a cytotoxic phenotype. Three major histologic patterns of skin involvement are recognized: epidermotropic, dermal, and predominantly subcutaneous. Often more than one histologic pattern is observed in the same skin biopsy. A hemophagocytic syndrome commonly occurs in patients with panniculitis-like tumors. Importantly, apoptosis and necrosis are common, often with angioinvasion. The tumor cells show a mature activated $\gamma / \delta$ cytotoxic T-cell phenotype with $\mathrm{CD} 3+\mathrm{CD} 2+\mathrm{CD} 56+$ and are negative for $\beta \mathrm{F} 1, \mathrm{CD} 4$, and usually $\mathrm{CD} 8$. The neoplastic cells show clonal rearrangement of TCR $\gamma$ and TCR $\delta$ genes, and EBV is negative. Patients with subcutaneous fat involvement tend to have a poor prognosis. An issue still not resolved is whether cutaneous and mucosal $\gamma / \delta$ TCL are part of the same disease.

Primary cutaneous CD8-positive aggressive epidermotropic cytotoxic T-cell lymphoma This is a new provisional entity in the 2008 WHO classification. This CTCL is characterized by a proliferation of epidermotropic CD8+ cytotoxic T-cells and a very aggressive clinical behavior. Patients present with disseminated eruptive papules, nodules, and tumors showing central ulceration and necrosis. One important feature of this CTCL is its unusual dissemination to visceral sites (lung, testis, central nervous system, and oral mucosa) without spreading to the lymph nodes. The tumor cells have a $\beta \mathrm{F} 1+, \mathrm{CD} 3+, \mathrm{CD} 8+$, and cytotoxic proteins + phenotype, with frequent loss of other T-cell antigens (CD2, CD5, CD7, and CD45RO). EBV is negative. The neoplastic cells show clonal TCR gene rearrangements, and specific genetic abnormalities have not been described.

Primary cutaneous CD4-positive small/medium T-cell lymphoma This is a new provisional entity in the 2008 WHO classification. This CTCL is characterized by a predominance of small to medium-sized CD4+ pleomorphic T-cells without features of MF and a very good prognosis. Most of the cases present as a solitary skin lesion. The lesions present usually on the head, neck, or upper trunk. By definition, these lymphomas have a CD3+, CD4+, CD8-, and CD30phenotype, sometimes with loss of pan-T-cell markers. Cytotoxic proteins are not expressed. Of note is that these lymphomas might show admixed plasma cells and B-cells, making the differential diagnosis from a reactive process difficult. However, the loss of pan-T-cell markers and/or clonal rearrangement of TCR genes are very useful criteria 
for the differential diagnosis. EBV is negative. No specific genetic abnormalities have been described.

Blastic plasmocytoid dendritic cell neoplasm

The last disease to be briefly mentioned herein is blastic plasmocytoid dendritic cell neoplasm. In the previous WHO classification, this entity was known as blastic NK-cell lymphoma. Blastic NK-cell lymphoma was described as a clinically aggressive neoplasm with a high incidence of cutaneous involvement and risk of leukemic dissemination. The blastic cytologic appearance and the CD56 expression initially suggested an NKprecursor origin. However, recent molecular and immunohistochemical studies suggested that these tumors derived from a plasmocytoid dendritic cell precursor.
Therefore, it was decided in the 2008 WHO to rename this entity and to include it in the group of myeloid neoplasias. The BPDC is a rare hematologic neoplasm with a predilection for the skin, followed by bone marrow and peripheral blood. Tumor cells express CD4, CD43, CD56, TCL1, and CD123. TdT is positive in a third of the cases. Because other CTCL may express CD4 and CD56, it is mandatory to perform an extensive immunohistochemical analysis before a definitive diagnosis of BPDC is made.

\section{References}

1. Swerdlow SH, Campo E, Harris NL, Jaffe ES, Pileri SA, Stein H, Thiele J, Vardiman JW (2008) Pathology and genetics of tumours of hematopoietic and lymphoid tissues, Chap. 11.270-299367. World Health Organization classification of tumours. International Agency for Research on Cancer Press, Lyon 